\title{
Unidirectional steady flow of a viscoelastic fluid with a free surface
}

\author{
José Miguel URBANO ${ }^{\dagger}$ \\ Departamento de Matemática, Universidade de Coimbra, Apartado 3008, 3001-454 Coimbra, \\ Portugal \\ AND \\ JUHA H. VIDEMAN ${ }^{\ddagger}$ \\ Departamento de Matemática, Instituto Superior Técnico, Av. Rovisco Pais, 1049-001 Lisboa, \\ Portugal
}

[Received 13 July 2000 and in revised form 31 January 2001]

\begin{abstract}
We study the steady flow of a second grade fluid down an open inclined channel. We formulate the mathematical problem, a mixed boundary value problem for the Laplacian with an unknown free boundary described by a nonlinear second-order ODE, and prove existence of a unique solution for small data using a contraction argument.
\end{abstract}

Keywords: Free boundary problems; second grade fluids; fixed point method

\section{Introduction}

The problem of a stationary flow of a viscous incompressible fluid with a free boundary has been the subject of numerous papers: see, for example, $[1,5,7,8,12,13]$ and references therein. For most of these works the method of reasoning to find the velocity field $v$ and the unknown free boundary $\phi$ can be reduced to the following steps: (1) find an equilibrium solution $\left(v_{0}, \phi_{0}\right)$, often corresponding to the 'zero data' solution; (2) linearize around $\left(v_{0}, \phi_{0}\right)$ and write the equations for the perturbation $(\tilde{v}, \tilde{\phi})$, i.e. $v=v_{0}+\tilde{v}, \phi=\phi_{0}+\tilde{\phi}$; (3) define a change of coordinates that transforms the domain with the free boundary $\Omega_{\phi}$ into a fixed domain $\Omega_{0}$ corresponding to the equilibrium solution and (4) solve (for small data) the coupled equations for the pair $(\tilde{v}, \tilde{\phi})$ in $\Omega_{0}$ by a fixed-point argument.

Here, we shall study a free boundary problem corresponding to the steady flow of an incompressible, homogeneous viscoelastic fluid down an inclined open infinite straight channel. The data of the problem are the inclination of the channel bed, the atmospheric pressure at the free boundary and the velocity flux over the channel bed cross section. For the model under consideration - the (Rivlin-Ericksen) fluid of second-grade - it makes sense to assume that the flow is rectilinear. Let us point out that also in a straight pipe with an arbitrary smooth cross section the equations of motion of the second-grade fluid admit solutions in which the velocity field is of Poiseuille type, cf. [9] and also [6,16]. The hypothesis that the flow is rectilinear leads at each cross section, say $\Omega_{\phi}$, to a coupled problem composed of a mixed boundary value problem for the Laplacian and of a nonlinear second-order ODE for the free boundary. This problem differs from the corresponding Navier-Stokes problem since additional nonlinear terms are present in the ODE.

†Email: jmurb@mat.uc.pt

*Email: videman@math.ist.utl.pt 


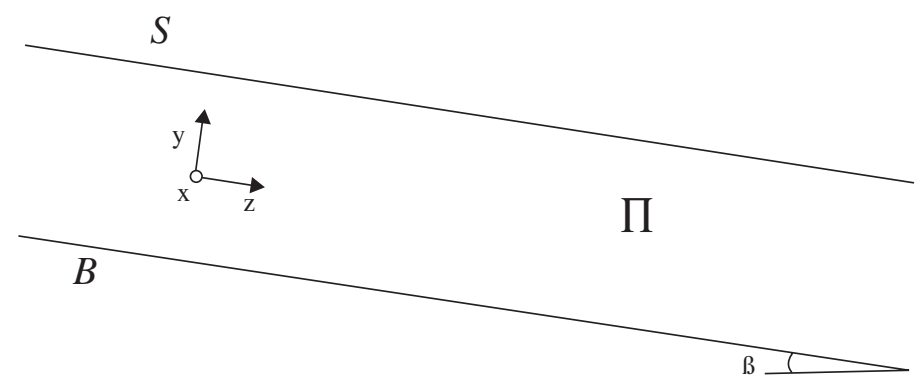

FIG. 1. Flow down an inclined channel.

Consequently, there is no hope, not even in the case when the channel bed is of a simple shape, of finding an (almost) explicit solution in an integral form as can be done for the Navier-Stokes fluid, cf. [4].

In this paper, we analyse the solvability of the coupled problem at each cross section $\Omega_{\phi}$ when the contact points where the fluid meets the channel bed are known, the case in which the flux condition becomes redundant. In a forthcoming work [15] we shall consider the problem with contact points that are not known a priori and show that for any given flux and for a small enough inclination of the channel bed, there exists a solution to the coupled set of equations that corresponds to a (unique) small perturbation of the equilibrium solution. Let us point out that in this case the transformation of coordinates becomes rather more complicated.

The paper is organized as follows. In Section 2, we introduce the physical formulation of the problem and present the corresponding mathematical model. In Section 3, we define and study the transformation of coordinates from $\Omega_{\phi}$ into a fixed domain $\Omega_{0}$. The main result, the existence of a unique solution for small data, is formulated and proven in Section 4 by means of a fixedpoint argument. Finally, Sections 5 and 6 are devoted to the study of a boundary value problem for the Laplacian and to the analysis of a nonlinear second-order ODE, respectively, which are the ingredients for the proof of the main theorem.

\section{The physical problem and the mathematical model}

Consider the flow of an incompressible Rivlin-Ericksen fluid of second grade down an infinite inclined open channel $\Pi$. Let us denote by $\mathcal{B}$ the wetted part of the channel bed and by $\mathcal{S}$ the fluid surface with unit outward normal vector $\mathbf{n}$. Assume that the channel bed forms an angle $\beta$ with the horizontal line and that the axis of the channel is parallel to the $z$-axis, see Fig. 1.

The equations of motion governing the stationary flow of an incompressible fluid are

$$
\rho \mathbf{v} \cdot \nabla \mathbf{v}+\nabla p=\rho \mathbf{f}+\nabla \cdot \mathbf{T}_{E}, \quad \nabla \cdot \mathbf{v}=0, \quad \text { in } \quad \Pi
$$

with $\mathbf{v}$ denoting the velocity field, $p$ the hydrostatic pressure, $\mathbf{T}_{E}$ the extra-stress tensor ( $\mathbf{T}=-p \mathbf{I}+\mathbf{T}_{E}$ is the usual Cauchy stress), $\mathbf{f}$ the external body force and $\rho$ the constant density of the fluid.

In an incompressible Rivlin-Ericksen fluid of second grade the extra-stress tensor $\mathbf{T}_{E}$ is related to kinematic variables by [10]

$$
\mathbf{T}_{E}=\eta \mathbf{A}_{1}(\mathbf{v})+\alpha_{1} \mathbf{A}_{2}(\mathbf{v})+\alpha_{2} \mathbf{A}_{1}^{2}(\mathbf{v}),
$$


where $\mathbf{A}_{1}(\mathbf{v})$ and $\mathbf{A}_{2}(\mathbf{v})$ denote the first two Rivlin-Ericksen tensors defined by

$$
\begin{aligned}
& \mathbf{A}_{1}(\mathbf{v})=\nabla \mathbf{v}+(\nabla \mathbf{v})^{T}, \\
& \mathbf{A}_{2}(\mathbf{v})=(\mathbf{v} \cdot \nabla) \mathbf{A}_{1}(\mathbf{v})+\mathbf{A}_{1}(\mathbf{v}) \nabla \mathbf{v}+(\nabla \mathbf{v})^{T} \mathbf{A}_{1}(\mathbf{v}),
\end{aligned}
$$

and $\eta, \alpha_{1}$ and $\alpha_{2}$ stand for material constants. For the second grade fluid to be consistent with thermodynamics, the material constants must satisfy, cf. [2],

$$
\eta \geqslant 0, \quad \alpha_{1} \geqslant 0, \quad \alpha_{1}+\alpha_{2}=0 .
$$

The constitutive relation (2) together with the equations (1) lead to the following set of equations for $(\mathbf{v}, p)$ :

$$
\left\{\begin{array}{l}
-\eta \Delta \mathbf{v}-\alpha_{1} \mathbf{v} \cdot \nabla \Delta \mathbf{v}+\nabla p=\rho \mathbf{f}+\nabla \cdot \mathbf{N}(\mathbf{v}) \quad \text { in } \quad \Pi \\
\nabla \cdot \mathbf{v}=0
\end{array}\right.
$$

with the nonlinear term $\mathbf{N}(\mathbf{v})$ given by

$$
\mathbf{N}(\mathbf{v})=\alpha_{1}(\nabla \mathbf{v})^{T} \mathbf{A}_{1}(\mathbf{v})-\rho \mathbf{v} \otimes \mathbf{v},
$$

and where we have taken into account the thermodynamical restriction $(4)_{3}$. As the boundary conditions are concerned, at the wetted part of the channel bed we assume the adherence condition

$$
\mathbf{v}=0 \quad \text { on } \quad \mathcal{B}
$$

and at the free surface $\mathcal{S}$ the usual kinematic and dynamic conditions, respectively (see, for example, [13])

$$
\mathbf{v} \cdot \mathbf{n}=0 \quad \text { and } \quad \mathbf{T n}-\sigma K \mathbf{n}=p_{0} \mathbf{n} \quad \text { on } \quad \mathcal{S},
$$

where $K=K(x, y, z)$ denotes the mean curvature of $\mathcal{S}, \sigma$ is the surface tension coefficient and $p_{0}$ a given exterior pressure. Finally, to complete the formulation (5)-(7), we impose the flux condition

$$
\int_{\Sigma} \mathbf{v} \mathrm{d} s=\Phi,
$$

where $\Sigma$ denotes an arbitrary cross section of $\Pi$ and $\Phi \in \mathbb{R}$ stands for a prescribed flux.

In an infinite straight cylinder with an arbitrary smooth cross section, the second-grade fluid equations (5), complemented with appropriate boundary and flux conditions, admit a solution having a flow pattern of Poiseuille type, cf. [9], so it is reasonable also here to look for a velocity field in the form

$$
\mathbf{v}=(0,0, w(x, y)) .
$$

Let $B$ and $\Gamma$ stand, respectively, for the intersection of the wetted part of the channel bed and the fluid surface with the $x y$-plane and suppose that the channel bed cross section is given by a smooth function $y=f(x)$, see Fig. 2. 


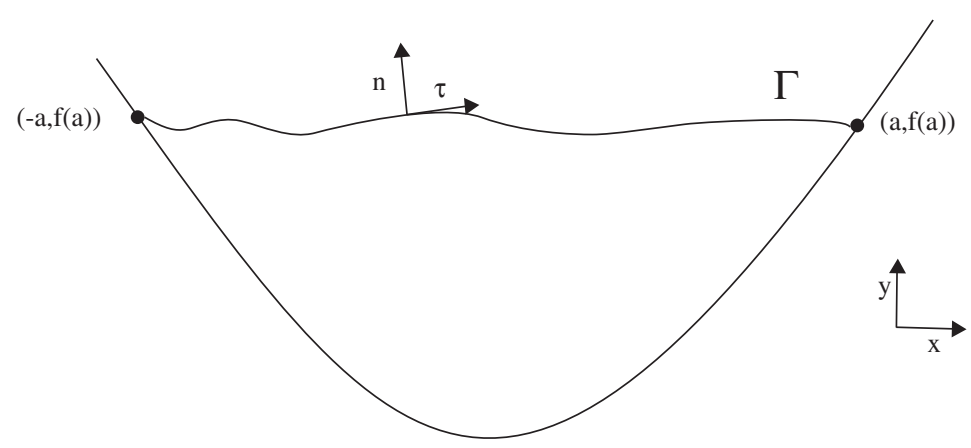

FIG. 2. Cross section of the channel.

Assume that the flow is driven (only) by the gravitational force, hence $\mathbf{f}=\nabla G$ with $G=g(-y \cos \beta+z \sin \beta)$, where $g$ is the (constant) acceleration of gravity and suppose, furthermore, that the pressure function has the form

$$
p=p(x, y)
$$

Substituting (9) and (10) into (5), yields

$$
\left\{\begin{array}{l}
\partial_{x} p=\frac{\alpha_{1}}{2} \partial_{x}\left|\nabla_{(x, y)} w\right|^{2}+\alpha_{1} \partial_{x} w \Delta_{(x, y)} w \\
\partial_{y} p=\frac{\alpha_{1}}{2} \partial_{y}\left|\nabla_{(x, y)} w\right|^{2}+\alpha_{1} \partial_{y} w \Delta_{(x, y)} w-\rho g \cos \beta \\
-\eta \Delta_{(x, y)} w=\rho g \sin \beta
\end{array}\right.
$$

and from (11) one readily obtains

$$
p=\frac{\alpha_{1}}{2}\left|\nabla_{(x, y)} w\right|^{2}+\frac{\alpha_{1} \rho g \sin \beta}{\eta} w-\rho g \cos \beta y+p_{c}
$$

where $p_{c} \in \mathbb{R}$.

Next, let us consider the boundary conditions. We denote by $\mathbf{n}=\left(n_{1}, n_{2}, 0\right)$ the unit normal vector to the free surface and choose a tangential vector of the form

$$
\tau=\frac{1}{\sqrt{2}}\left(n_{2},-n_{1}, 1\right)
$$

Taking the scalar product of $(7)_{2}$ with $\tau$, one easily obtains the following Neumann condition for $w$ :

$$
\frac{\partial w}{\partial n}=\frac{\alpha_{1}}{\eta}\left(\left(\left(\partial_{y} w\right)^{2}-\left(\partial_{x} w\right)^{2}\right) n_{1} n_{2}+\partial_{x} w \partial_{y} w\left(n_{1}^{2}-n_{2}^{2}\right)\right)=-\sqrt{2} \frac{\alpha_{1}}{\eta} \frac{\partial w}{\partial n} \frac{\partial w}{\partial \tau} \quad \text { on } \quad \Gamma
$$

that naturally leads to the homogeneous condition $\frac{\partial w}{\partial n}=0$ on $\Gamma$. Observe also that $(7)_{1}$ is automatically satisfied and that condition (6) reduces to

$$
w=0 \text { on } B .
$$


On the other hand, taking the scalar product of $(7)_{2}$ with $\mathbf{n}$, we obtain the equation for the unknown free surface $\mathcal{S}$

$$
\sigma K(x, y, z)=\left.\left(-p+\mathbf{n} \cdot \mathbf{T}_{E} \mathbf{n}-p_{0}\right)\right|_{\mathcal{S}} .
$$

Assuming that the free boundary $\Gamma$ at the channel bed cross section is described by $y=\phi(x)$, condition (13) takes the form of the following ordinary differential equation for $\phi(x)$ :

$$
\sigma \frac{\phi^{\prime \prime}}{\left(1+\phi^{\prime 2}\right)^{\frac{3}{2}}}=\left.\left(\alpha_{1}\left|\frac{\partial w}{\partial n}\right|^{2}-p-p_{0}\right)\right|_{y=\phi(x)} .
$$

(Observe that here we have tacitly assumed that the fluid stays always below the surface $\Gamma$.) The boundary conditions for (14) are obtained by assuming that the fluid surface cross section $\Gamma$ and the wetted channel bed cross section $B$ meet at the contact points $(-a, f(-a))$ and $(a, f(a))$, with $f(-a)=f(a)$. Note that, by fixing the contact points, we have made the flux condition (8) redundant and, in fact, it will be absent from our formulation.

Now, at the free boundary given by $y=\phi(x)$, one may write

$$
\mathbf{n}=\frac{1}{\sqrt{1+\phi^{\prime 2}}}\left(-\phi^{\prime}, 1,0\right),
$$

and, hence, the problem is to determine $(\phi, w)$ satisfying

$$
\left\{\begin{array}{lll}
-\Delta w=\frac{\rho g \sin \beta}{\eta} & \text { in } & \Omega_{\phi} \\
w=0 & \text { on } & B \\
\partial w / \partial n=0 & \text { on } & \phi \\
\frac{\phi^{\prime \prime}}{\left(1+\phi^{\prime 2}\right)^{\frac{3}{2}}}=h\left(w_{\phi}, w_{\phi}, \phi\right) & \text { on } & (-a, a) \\
\phi(-a)=\phi(a)=f(a) & &
\end{array}\right.
$$

with

$$
\begin{aligned}
\Omega_{\psi} & =\left\{(x, y) \in \mathbb{R}^{2}:-a<x<a, f(x)<y<\psi(x)\right\}, \\
h(u, v, \psi) & =-\frac{\alpha_{1}}{2 \sigma}\left|\nabla u_{\psi}\right|^{2}-\frac{\alpha_{1} \rho g \sin \beta}{\sigma \eta} v_{\psi}+\frac{\rho g \cos \beta}{\sigma} \psi-\frac{\rho g \cos \beta}{\sigma} p_{0}, \\
u_{\psi} & =u(x, \psi(x)),
\end{aligned}
$$

where we have redefined $p_{0}$ and dropped the subscripts $\cdot(x, y)$ since all the functions depend at most on $x$ and $y$.

\section{The transformation of coordinates}

We shall show in Section 4 that, for small data, problem (15) admits a unique solution. The proof is achieved using Banach's fixed point theorem and an essential ingredient are estimates that require the comparison of functions. The functions to be compared must be defined in the same domain and, hence, we are led to introduce the reference domain

$$
\Omega_{0}=\left\{\left(z_{1}, z_{2}\right) \in \mathbb{R}^{2}:-a<z_{1}<a, f\left(z_{1}\right)<z_{2}<f(a)\right\} .
$$


Now, using a transformation of coordinates $\mathcal{F}_{\phi}: \Omega_{\phi} \rightarrow \Omega_{0}$ defined by the expression

$$
(x, y) \longmapsto\left(z_{1}, z_{2}\right)=\left(x, \frac{f(a)-f(x)}{\phi(x)-f(x)}(y-f(x))+f(x)\right),
$$

we can shift to $\Omega_{0}$ from any domain of the type $\Omega_{\phi}$. In order to simplify the computations that follow let us introduce the functions, defined in the interval $(-a, a)$,

$$
s(\xi)=\frac{f(a)-f(\xi)}{\phi(\xi)-f(\xi)}
$$

and

so that

$$
q(\xi)=\frac{\phi(\xi)-f(a)}{\phi(\xi)-f(\xi)} f(\xi)
$$

and, by inversion,

$$
z_{1}=x, \quad z_{2}=s(x) y+q(x)
$$

$$
x=z_{1}, \quad y=\frac{1}{s\left(z_{1}\right)}\left[z_{2}-q\left(z_{1}\right)\right] .
$$

It will also be useful in the sequel to work with the function defined in $\Omega_{0}$ by

$$
r\left(\xi_{1}, \xi_{2}\right)=\frac{s^{\prime}\left(\xi_{1}\right)}{s\left(\xi_{1}\right)}\left[\xi_{2}-q\left(\xi_{1}\right)\right]+q^{\prime}\left(\xi_{1}\right) .
$$

From now on, we assume that the function $y=f(x)$ that defines the channel bed is regular and symmetric with respect to the $y$-axis, monotone increasing for $x>0$ and such that $f(0)=0$. Furthermore, we assume that

$$
0 \leqslant f(x) \leqslant \frac{f(a)}{a^{2}} x^{2}, \quad \forall x \in[-a, a] .
$$

LEMmA 3.1 Given $\phi \in C^{2}[-a, a]$ such that $\phi(a)=\phi(-a)=f(a)$ and

$$
\|\phi-f(a)\|_{C^{0}}+\left\|\phi^{\prime}\right\|_{C^{1}} \leqslant \frac{f(a)}{2 a},
$$

the functions $s$ and $r$ defined by (18) and (19) are, respectively, of class $C^{2}[-a, a]$ and $C^{1}\left(\overline{\Omega_{0}}\right)$ (after being appropriately defined at $\pm a$ and $\partial \Omega_{0}$ ). Moreover, it holds that

$$
\|s\|_{C^{2}}+\|r\|_{C^{1}} \leqslant c\|f\|_{C^{2}} \text {. }
$$

Proof. Both functions $s$ and $r$ can be shown to be well defined at $\xi_{1}= \pm a$ and $\left(\xi_{1}, \xi_{2}\right)=$ ( $\pm a, f(a)$ ) respectively, together with some of their derivatives (it is obvious how to extend $r$ to the other points in $\partial \Omega_{0}$ ). Let us show this, for example, for $r$ and $s$; the case of the derivatives is similar, although somehow more technical. Letting $m\left(\xi_{1}\right)=f(a)-f\left(\xi_{1}\right)$ and $n\left(\xi_{1}\right)=\phi\left(\xi_{1}\right)-f\left(\xi_{1}\right)$, we can write

$$
\begin{aligned}
r & =\frac{m^{\prime} n-m n^{\prime}}{m n}\left[\xi_{2}+f\left(\frac{m}{n}-1\right)\right]-f^{\prime}\left(\frac{m}{n}-1\right)-f\left(\frac{m^{\prime} n-m n^{\prime}}{n^{2}}\right) \\
& =\left(\frac{m^{\prime}}{m}-\frac{n^{\prime}}{n}\right)\left(\xi_{2}-f\right)-f^{\prime}\left(\frac{m}{n}-1\right)
\end{aligned}
$$


and so in the limit we get

$$
\lim _{\substack{\xi_{1} \rightarrow a \\ \xi_{2} \rightarrow f(a)}} r\left(\xi_{1}, \xi_{2}\right)=\frac{\phi^{\prime}(a) f^{\prime}(a)}{\phi^{\prime}(a)-f^{\prime}(a)}
$$

For $s$ one easily gets

$$
\lim _{\xi_{1} \rightarrow a} s\left(\xi_{1}\right)=\frac{f^{\prime}(a)}{f^{\prime}(a)-\phi^{\prime}(a)} .
$$

Concerning the interior, assumption (20) implies in particular that

$$
\phi\left(\xi_{1}\right)-f\left(\xi_{1}\right) \geqslant \frac{f(a)}{2 a^{2}}\left(a-\xi_{1}\right)\left(a+2 \xi_{1}\right), \quad \forall \xi_{1} \in(0, a) .
$$

Hence, observing that since from (20) it also follows that

$$
\left|f^{\prime}(a)\right| \geqslant 2 \frac{f(a)}{a},
$$

one has $\left|f^{\prime}(a)-\phi^{\prime}(a)\right| \leqslant \frac{3 f(a)}{2}$ and concludes, after a simple computation, that

$$
\left|s\left(\xi_{1}\right)\right| \leqslant 2 a \frac{f^{\prime}(a)}{f(a)}, \quad \forall \xi_{1} \in[0, a] .
$$

A similar estimate can be obtained in $[-a, 0]$. On the other hand, it is easy to see that

$$
f(a)-\xi_{2} \leqslant\left(a-\xi_{1}\right)\left|f^{\prime}( \pm a)\right|, \quad 0<\xi_{1}<a, f\left(\xi_{1}\right)<\xi_{2}<f(a)
$$

and similarly in the other half of $\Omega_{0}$. Using this, together with (20), in (19) yields the estimate

$$
\max _{\left(\xi_{1}, \xi_{2}\right) \in \Omega_{0}}\left|r\left(\xi_{1}, \xi_{2}\right)\right| \leqslant c\left(\frac{\left[f^{\prime}(a)\right]^{2}}{f(a)}+\left|f^{\prime}(a)\right|\right)
$$

where we have also taken into account that

$$
\left|f^{\prime}\left(\xi_{1}\right)\right| \leqslant\left|f^{\prime}( \pm a)\right|, \quad \forall \xi_{1} \in[-a, a] .
$$

The derivatives of $s$ and $r$ can be estimated analogously.

\section{The existence result}

In this section we prove our main theorem, namely the existence of a unique solution to problem (15) under the assumptions stated below.

(A1) $f$ is a regular, even function such that $f(0)=0, f^{\prime}(x)>0$, for $x>0$, and (20) holds.

(A2) $p_{0}=f(a)+\delta, \delta \ll 1$.

(A3) $\exists \beta_{0}>0, \beta_{0} \ll 1: 0<|\beta| \leqslant \beta_{0}<\frac{\pi}{2}$. 
THEOREM 4.1 Under assumptions (A1)-(A3), problem (15) admits a unique solution $(\phi, w) \in$ $C^{2}[-a, a] \times W^{2, p}\left(\Omega_{\phi}\right)$, provided $p>2$ is such that

$$
\tan \left(\frac{\pi p}{4(p-1)}\right)> \pm f^{\prime}( \pm a)
$$

Proof. We apply the contraction mapping principle of Banach to an operator $\mathcal{T}: X \times Y \longrightarrow X \times Y$, where the Banach spaces $X$ and $Y$ are

$$
X=\left\{\tilde{\psi} \in C^{2}[-a, a]: \tilde{\psi}( \pm a)=0\right\}
$$

and $Y=W^{2, p}\left(\Omega_{0}\right)$. We define a closed ball in $X \times Y$ by

$$
\mathbb{B}_{\varrho}=\left\{(\tilde{\psi}, u) \in X \times Y:\|\tilde{\psi}\|_{C^{2}}+\|u\|_{2, p} \leqslant \varrho\right\} .
$$

The operator $\mathcal{T}$ is defined as follows. Given $(\tilde{\psi}, u) \in X \times Y$, let $\psi=\tilde{\psi}+f(a)$ and consider the transformation of coordinates $\mathcal{F}_{\psi}$ associated with $\psi$ and defined in the previous section by (17). The boundary value problem

$$
\left\{\begin{array}{llc}
-\Delta \hat{u}=\frac{\rho g \sin \beta}{\eta} & \text { in } \quad \Omega_{\psi} \\
\hat{u}=0 & \text { on } \quad B \\
\frac{\partial \hat{u}}{\partial n}=0 & \text { on } \quad \psi
\end{array}\right.
$$

is transformed, using $\mathcal{F}_{\psi}$ and $\mathcal{F}_{\psi}^{-1}$, into the following problem in $\Omega_{0}$ :

$$
\left\{\begin{array}{lll}
\mathcal{A} v=\frac{\rho g \sin \beta}{\eta} & \text { in } & \Omega_{0} \\
v=0 & \text { on } & B \\
\mathcal{N} v=0 & \text { on } & \Gamma_{0} .
\end{array}\right.
$$

To the free boundary now corresponds the straight line

$$
\Gamma_{0}=\left\{\left(z_{1}, z_{2}\right):-a<z_{1}<a, z_{2}=f(a)\right\}
$$

and the new differential operators are given by

$$
\mathcal{A} v=-\left[\frac{\partial^{2} v}{\partial z_{1}^{2}}+\left(r^{2}+s^{2}\right) \frac{\partial^{2} v}{\partial z_{2}^{2}}\right]-2 r \frac{\partial^{2} v}{\partial z_{1} \partial z_{2}}-\left(\frac{\partial r}{\partial z_{1}}+r \frac{\partial r}{\partial z_{2}}\right) \frac{\partial v}{\partial z_{2}}
$$

and

$$
\mathcal{N} v=\left(1+\psi^{\prime 2}\right)^{-\frac{1}{2}}\left[-\psi^{\prime} \frac{\partial v}{\partial z_{1}}+\left(s-\psi^{\prime} r\right) \frac{\partial v}{\partial z_{2}}\right]
$$

where $s$ and $r$, that are defined in (18) and (19), obviously depend on $\psi$. In fact, observe that the derivatives read, in new coordinates,

$$
\frac{\partial}{\partial x}=\frac{\partial}{\partial z_{1}} \frac{\partial z_{1}}{\partial x}+\frac{\partial}{\partial z_{2}} \frac{\partial z_{2}}{\partial x}=\frac{\partial}{\partial z_{1}}+r\left(z_{1}, z_{2}\right) \frac{\partial}{\partial z_{2}}
$$


and

$$
\frac{\partial}{\partial y}=\frac{\partial}{\partial z_{2}} \frac{\partial z_{2}}{\partial y}=s\left(z_{1}\right) \frac{\partial}{\partial z_{2}}
$$

and the second derivatives

$$
\frac{\partial^{2}}{\partial x^{2}}=\frac{\partial^{2}}{\partial z_{1}^{2}}+\left(\frac{\partial r}{\partial z_{1}}+r \frac{\partial r}{\partial z_{2}}\right) \frac{\partial}{\partial z_{2}}+2 r \frac{\partial^{2}}{\partial z_{1} \partial z_{2}}+r^{2} \frac{\partial^{2}}{\partial z_{2}^{2}}
$$

and

$$
\frac{\partial^{2}}{\partial^{2} y}=s^{2} \frac{\partial^{2}}{\partial z_{2}^{2}} .
$$

We solve problem (26) in Section 5 (cf. Lemma 5.1) and obtain a unique function $v \in W^{2, p}\left(\Omega_{0}\right)$. Note that condition (29) in Lemma 5.1 can be satisfied for sufficiently small $\varrho$ in view of assumption (24). It is obvious that $v=\hat{u} \circ \mathcal{F}_{\psi}^{-1}$, i.e. $\hat{u}=v \circ \mathcal{F}_{\psi}$.

With $v$ at hand, we consider the ODE for $\varphi$ (recall the definition of $h$ from Section 2)

$$
\frac{\varphi^{\prime \prime}}{\left(1+\varphi^{\prime 2}\right)^{3 / 2}}=h\left(u \circ \mathcal{F}_{\psi}, v \circ \mathcal{F}_{\psi}, \psi\right)
$$

with the boundary conditions

$$
\varphi(-a)=\varphi(a)=f(a)
$$

and solve it in Section 6 (cf. Lemma 6.1) obtaining a unique solution $\varphi \in C^{2}[-a, a]$.

Next, we set $\tilde{\varphi}=\varphi-f(a)$ and finally define the mapping $\mathcal{T}$ by

$$
\mathcal{T}(\tilde{\psi}, u)=(\tilde{\varphi}, v) .
$$

It is apparent that the definition is unambiguous. On the other hand, from estimates (30) and (38), see Lemmas 5.1 and 6.1, it follows that $\mathcal{T}$ maps the ball $\mathbb{B}_{\varrho}$ into itself, provided $\beta_{0}, \delta, \varrho \ll 1$ are chosen small enough. Moreover, again for small enough $\beta_{0}, \delta$ and $\varrho$, Lemmas 5.2 and 6.2 guarantee the existence of a constant $\gamma<1$ such that

$$
\left\|\mathcal{T}\left(\tilde{\psi}_{1}, u_{1}\right)-\mathcal{T}\left(\tilde{\psi}_{2}, u_{2}\right)\right\|_{X \times Y} \leqslant \gamma\left\|\left(\tilde{\psi}_{1}, u_{1}\right)-\left(\tilde{\psi}_{2}, u_{2}\right)\right\|_{X \times Y} .
$$

Therefore, one concludes from Banach's contraction principle that $\mathcal{T}$ possesses a unique fixed-point $\mathcal{T}\left(\tilde{\psi}^{*}, u^{*}\right)=\left(\tilde{\psi}^{*}, u^{*}\right)$. Then the pair

$$
(\phi, w)=\left(\tilde{\psi}^{*}+f(a), u^{*} \circ \mathcal{F}_{\left(\tilde{\psi}^{*}+f(a)\right)}\right)
$$

is the unique solution of problem (15).

\section{Analysis of the BVP}

In this section we analyse the boundary value problem (26) and prove the existence and uniqueness of a weak solution and establish the estimates needed for the use of the fixed-point theorem. 
LEMMA 5.1 Given $u \in W^{2, p}\left(\Omega_{0}\right)$ and $\phi \in C^{2}[-a, a]$ satisfying the assumptions of Lemma 3.1, problem (26) (with $\phi$ in place of $\psi$ ) admits a unique solution $v \in W^{2, p}\left(\Omega_{0}\right)$ provided

$$
\phi^{\prime}( \pm a) \gtrless \frac{f^{\prime}( \pm a) \mp \tan \left(\frac{\pi p}{4(p-1)}\right)}{1 \pm f^{\prime}( \pm a) \tan \left(\frac{\pi p}{4(p-1)}\right)} .
$$

Moreover, the following estimate holds:

$$
\|v\|_{2, p} \leqslant c \sin \beta_{0}
$$

REMARK 5.1 Note that with $\phi$ we define $s$ and $r$ in (26) through (18) and (19).

Proof. To show that the problem has a unique solution, we use well known results from elliptic theory [3]. Observe that the operator $\mathcal{A}$ can be written in the form

$$
\mathcal{A} v=\sum_{i, j=1}^{2} \frac{\partial}{\partial z_{i}}\left(a_{i, j} \frac{\partial v}{\partial z_{j}}\right)+\sum_{i=1}^{2} a_{i} \frac{\partial v}{\partial z_{i}}
$$

with

$$
\left\{\begin{array} { l } 
{ a _ { 1 , 1 } = - 1 } \\
{ a _ { 1 , 2 } = a _ { 2 , 1 } = - r } \\
{ a _ { 2 , 2 } = - r ^ { 2 } - s ^ { 2 } }
\end{array} \quad \text { and } \quad \left\{\begin{array}{l}
a_{1}=\frac{\partial r}{\partial z_{2}} \\
a_{2}=r \frac{\partial r}{\partial z_{2}} .
\end{array}\right.\right.
$$

The operator is strongly elliptic since, for a certain $\alpha>0$,

$$
a_{1,1} \xi_{1}^{2}+2 a_{1,2} \xi_{1} \xi_{2}+a_{2,2} \xi_{2}^{2}<-\alpha|\xi|^{2}, \quad \forall \xi=\left(\xi_{1}, \xi_{2}\right) \in \mathbb{R}^{2} \backslash\{0\} .
$$

In fact, this is equivalent to

$$
\left(\xi_{1}+r \xi_{2}\right)^{2}+s^{2} \xi_{2}^{2}>\alpha\left(\xi_{1}^{2}+\xi_{2}^{2}\right)
$$

and dividing by $\xi_{1}^{2}$ (the case $\xi_{1}=0$ is trivial) and putting $\zeta=\xi_{2} / \xi_{1}$, to

$$
\left(r^{2}+s^{2}-\alpha\right) \zeta^{2}+2 r \zeta+(1-\alpha)>0, \forall \zeta \neq 0 .
$$

Standard computations show that this holds provided

$$
\alpha \leqslant r^{2}+s^{2} \quad \text { and } \quad \alpha^{2}-\left(r^{2}+s^{2}+1\right) \alpha+s^{2}<0
$$

and a sufficient condition is that

$$
\exists A, B, C>0: A \leqslant s^{2} \leqslant B \text { and } r^{2} \leqslant C .
$$

We clearly see that these conditions hold due to (23), the fact that a similar conclusion is valid for $z_{1} \rightarrow-a$, and the remark that the continuous function $s^{2}>0$ (it is obvious that it never vanishes), properly redefined at $z_{1}=-a$ and $z_{1}=a$, attains its minimum $A>0$ and maximum $B>0$ in the compact $[-a, a]$. A reasoning of this type (using (22)) can also be made to conclude for the existence of $C>0$ such that $r^{2} \leqslant C$.

We could now apply classical results from elliptic theory to obtain existence and uniqueness of a solution $v \in W^{2, p}\left(\Omega_{0}\right)$ if it were not for the lack of smoothness at the contact points $p_{a}=(a, f(a))$ 
and $p_{-a}=(-a, f(a))$. To overcome this difficulty, we consider the problem in a neighbourhood of, say, $p_{a}$

$$
\Omega_{\delta}=\Omega_{0} \cap \mathcal{V}\left(p_{a}, \delta\right), \quad 0<\delta \ll 1 .
$$

We linearize problem (26) by freezing the coefficients of the operator $\mathcal{A}$ and $\mathcal{N}$ and neglect the terms containing the lower-order derivatives. The freezing is performed by taking the limit of the coefficients as $p_{a}$ is approached. Owing to (22) and (23) this leads to the linear operators

$$
\mathcal{A}_{0} \equiv-\frac{\partial^{2}}{\partial z_{1}^{2}}-\kappa_{1} \frac{\partial^{2}}{\partial z_{2}^{2}}-2 \kappa_{2} \frac{\partial^{2}}{\partial z_{1} \partial z_{2}}
$$

and

$$
\mathcal{N}_{0} \equiv-\phi^{\prime}(a)\left(1+\left[\phi^{\prime}(a)\right]^{2}\right)^{-\frac{1}{2}} \frac{\partial}{\partial z_{1}}+\sqrt{\kappa_{1}} \frac{\partial}{\partial z_{2}}
$$

where

$$
\kappa_{1}=\frac{\left[f^{\prime}(a)\right]^{2}\left(\left[\phi^{\prime}(a)\right]^{2}+1\right)}{\left[f^{\prime}(a)-\phi^{\prime}(a)\right]^{2}} \quad \text { and } \quad \kappa_{2}=\frac{f^{\prime}(a) \phi^{\prime}(a)}{\phi^{\prime}(a)-f^{\prime}(a)} .
$$

We next perform a change of variables defined by

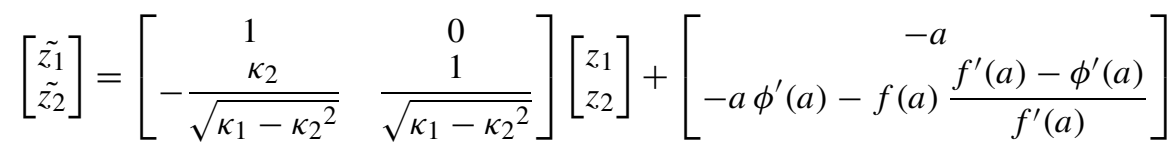

and obtain in the new coordinates $\left(\tilde{z_{1}}, \tilde{z_{2}}\right)$, taking into account that

$$
-\frac{\kappa_{2}}{\sqrt{\kappa_{1}-\kappa_{2}^{2}}}=\phi^{\prime}(a)
$$

a problem for the Laplace operator in $\widetilde{\Omega}_{\delta}$, where

$$
\begin{aligned}
\widetilde{\Omega_{\delta}}= & \left\{\left(\tilde{z_{1}}, \tilde{z_{2}}\right):-\delta<\tilde{z_{1}}<0,\right. \\
& \left.\phi^{\prime}(a) \tilde{z_{1}}+\frac{f^{\prime}(a)-\phi^{\prime}(a)}{f^{\prime}(a)}\left(f\left(\tilde{z_{1}}+a\right)-f(a)\right)<\tilde{z_{2}}<\phi^{\prime}(a) \tilde{z_{1}}\right\} .
\end{aligned}
$$

Note that the origin of coordinates is now placed on $p_{a}$. As far as the differential operator $\mathcal{N}_{0}$ on the boundary is concerned, in view of the relations

$$
\begin{aligned}
& \frac{\partial}{\partial z_{1}}=\frac{\partial}{\partial \tilde{z_{1}}+\phi^{\prime}(a) \frac{\partial}{\partial \tilde{z_{2}}}} \\
& \frac{\partial}{\partial z_{2}}=\frac{f^{\prime}(a)}{f^{\prime}(a)-\phi^{\prime}(a)} \frac{\partial}{\partial \tilde{z_{2}}},
\end{aligned}
$$

one has in the new coordinates

$$
\tilde{\mathcal{N}}_{0} \tilde{u}=\frac{1}{\sqrt{1+\phi^{\prime 2}(a)}}\left(-\phi^{\prime}(a) \frac{\partial \tilde{u}}{\partial \tilde{z}_{1}}+\frac{\partial \tilde{u}}{\partial \tilde{z}_{2}}\right) .
$$


Since, in the neighbourhood of $p_{a}, \Gamma_{0}$ now corresponds to the line

$$
\widetilde{\Gamma_{0}}:=\left\{\left(\tilde{z_{1}}, \tilde{z_{2}}\right): \tilde{z_{2}}=\phi^{\prime}(a) \tilde{z_{1}}\right\},
$$

one easily sees that $\widetilde{\mathcal{N}_{0}} \tilde{u}=\nabla_{\left(\tilde{z_{1}}, \tilde{z_{2}}\right)} \tilde{u} \cdot \tilde{\mathbf{n}}$ on $\widetilde{\Gamma}_{0}$, where

$$
\tilde{\mathbf{n}}=\frac{1}{\sqrt{1+\phi^{\prime 2}(a)}}\left(-\phi^{\prime}(a), 1\right)
$$

is the unit normal vector on $\widetilde{\Gamma}_{0}$. Hence, the problem reduces to a Dirichlet-Neumann problem for the Laplacian and one can use the results of [3] (see, in particular, Corollary 4.4.3.8) to conclude that this problem has a unique $W^{2, p}$-solution provided the angle $\tilde{\omega}$ at the contact point in $\widetilde{\Omega_{\delta}}$ satisfies the relation

$$
\tilde{\omega}<\frac{\pi p}{4(p-1)}
$$

On the other hand, since

$$
\lim _{\tilde{z_{1}} \rightarrow 0} \frac{\mathrm{d}}{\mathrm{d} \tilde{z_{1}}}\left[\phi^{\prime}(a) \tilde{z_{1}}+\frac{f^{\prime}(a)-\phi^{\prime}(a)}{f^{\prime}(a)}\left(f\left(\tilde{z_{1}}+a\right)-f(a)\right)\right]=f^{\prime}(a),
$$

one concludes that the change of variables (31) transforms the contact angle $\omega=\arctan f^{\prime}(a)$ in $\Omega_{\delta}$ into the original contact angle $\arctan f^{\prime}(a)-\arctan \phi^{\prime}(a)$ in $\Omega$. Thus, we get

$$
\tilde{\omega}=\arctan f^{\prime}(a)-\arctan \phi^{\prime}(a)
$$

and using this in (32) we obtain condition (29) at $a$. Similarly, one obtains the condition at $-a$.

Finally, estimate (30) can be proven directly.

In the next result we estimate the norm of the difference of two solutions in terms of the differences of the data. Note that it is possible to choose $c<1$ if the data is small.

LemMA 5.2 Let $v_{i} \in W^{2, p}\left(\Omega_{0}\right), i=1,2$ denote two solutions of (26) corresponding to $\phi_{i} \in$ $C^{2}[-a, a], i=1,2$, respectively. Moreover, assume that $\phi_{i}, i=1,2$ satisfy the assumptions of Lemma 3.1. Then the following estimate holds:

$$
\left\|v_{1}-v_{2}\right\|_{2, p} \leqslant c\left\|\phi_{1}-\phi_{2}\right\|_{C^{2}},
$$

for some positive constant $c=c\left(\beta_{0},\|f\|_{C^{2}}\right)$.

Proof. Let $s_{i}$ and $r_{i}$ be the auxiliary functions (see definitions (18) and (19)) associated with $\phi_{i}$, $i=1,2$. The difference $v=v_{1}-v_{2}$ satisfies the following boundary value problem:

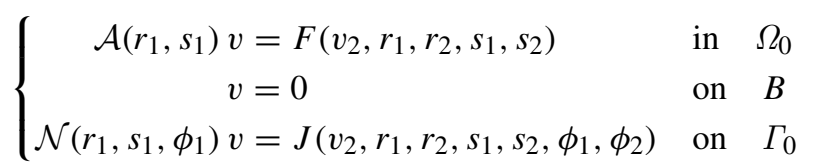


where

$$
\begin{aligned}
F\left(v_{2}, r_{1}, r_{2}, s_{1}, s_{2}\right)= & -\left(r\left(r_{1}+r_{2}\right)+s\left(s_{1}+s_{2}\right)\right) \frac{\partial^{2} v_{2}}{\partial z_{2}^{2}}+2 r \frac{\partial^{2} v_{2}}{\partial z_{1} \partial z_{2}} \\
& +\left(\frac{\partial r}{\partial z_{1}}+r \frac{\partial r_{1}}{\partial z_{2}}+r_{2} \frac{\partial r}{\partial z_{2}}\right) \frac{\partial v_{2}}{\partial z_{2}}
\end{aligned}
$$

and

$$
J\left(v_{2}, r_{1}, r_{2}, s_{1}, s_{2}, \phi_{1}, \phi_{2}\right)=\mathcal{N}\left(r_{2}, s_{2}, \phi_{2}\right) v_{2}-\mathcal{N}\left(r_{1}, s_{1}, \phi_{1}\right) v_{2}
$$

with $r=r_{1}-r_{2}$ and $s=s_{1}-s_{2}$. Taking the proof of Lemma 5.1 and the results of [3] into account, we are left to prove that $F \in L^{p}\left(\Omega_{0}\right)$ and $J \in W^{1-\frac{1}{p}, p}\left(\Gamma_{0}\right)$.

We start with the differences $s=s_{1}-s_{2}$ and $r=r_{1}-r_{2}$. One may write them in the form

$$
\begin{aligned}
& r=\frac{n_{2}\left(n_{1}^{\prime}-n_{2}^{\prime}\right)-\left(n_{1}-n_{2}\right) n_{2}^{\prime}}{n_{1} n_{2}}\left(f-z_{2}\right)+f^{\prime} m \frac{n_{1}-n_{2}}{n_{1} n_{2}} \\
& s=m \frac{n_{2}-n_{1}}{n_{1} n_{2}}=s_{1} s_{2} \frac{\phi_{2}-\phi_{1}}{m},
\end{aligned}
$$

where $m\left(z_{1}\right)=f(a)-f\left(z_{1}\right), i=1,2$ and $n_{i}\left(z_{1}\right)=\phi_{i}\left(z_{1}\right)-f\left(z_{1}\right), i=1,2$. One readily obtains the estimate

$$
\max _{z_{1} \in[-a, a]}\left|\frac{\phi_{2}\left(z_{1}\right)-\phi_{1}\left(z_{1}\right)}{m\left(z_{1}\right)}\right| \leqslant \frac{a}{f(a)}\left\|\phi_{2}^{\prime}-\phi_{1}^{\prime}\right\|_{C^{0}}
$$

and concludes that

$$
\begin{aligned}
\max _{\left(z_{1}, z_{2}\right) \in \Omega_{0}}\left|r\left(z_{1}, z_{2}\right)\right| & \leqslant c \frac{\left|f^{\prime}(a)\right|^{2}}{[f(a)]^{2}}\left(1+\frac{\left|f^{\prime}(a)\right|}{f(a)}\right)^{2}\left\|\phi_{2}^{\prime}-\phi_{1}^{\prime}\right\|_{C^{0}} \\
\max _{z_{1} \in[-a, a]}\left|s\left(z_{1}\right)\right| & \leqslant c \frac{\left|f^{\prime}(a)\right|}{f(a)^{2}}\left(1+\frac{\left|f^{\prime}(a)\right|}{f(a)}\right)\left\|\phi_{2}^{\prime}-\phi_{1}^{\prime}\right\|_{C^{0}} .
\end{aligned}
$$

We have used Lemma 3.1 and, in particular, the fact that

$$
\begin{aligned}
\lim _{z_{1} \rightarrow a} s\left(z_{1}\right) & =-\frac{f^{\prime}(a)\left(\phi_{2}^{\prime}(a)-\phi_{1}^{\prime}(a)\right)}{\left(\phi_{2}^{\prime}(a)-f^{\prime}(a)\right)\left(\phi_{1}^{\prime}(a)-f^{\prime}(a)\right)} \\
\lim _{\substack{z_{1} \rightarrow a \\
z_{2} \rightarrow f(a)}} r\left(z_{1}, z_{2}\right) & =\frac{\left[f^{\prime}(a)\right]^{2}\left(\phi_{2}^{\prime}(a)-\phi_{1}^{\prime}(a)\right)}{\left(\phi_{2}^{\prime}(a)-f^{\prime}(a)\right)\left(\phi_{1}^{\prime}(a)-f^{\prime}(a)\right)} .
\end{aligned}
$$

Estimating the derivatives of $s$ and $r$ in a similar fashion leads to

$$
\|s\|_{C^{1}}+\|r\|_{C^{1}} \leqslant c\left\|\phi_{2}^{\prime}-\phi_{1}^{\prime}\right\|_{C^{1}}, \quad c=c\left(\|f\|_{C^{2}}\right) .
$$

Since by Sobolev embeddings it holds $u_{i} \in C^{1}\left(\Omega_{0}\right), i=1,2$, one obtains the estimates

$$
\left\|F\left(v_{2}, r_{1}, r_{2}, s_{1}, s_{2}\right)\right\|_{p} \leqslant c\left\|v_{2}\right\|_{2, p}\left\|\phi_{1}-\phi_{2}\right\|_{C^{2}}
$$


and

$$
\begin{aligned}
& \left\|J\left(v_{2}, r_{1}, r_{2}, s_{1}, s_{2}, \phi_{1}, \phi_{2}\right)\right\|_{1-\frac{1}{p}, p, \Gamma_{0}} \\
& \quad \leqslant c\left\|v_{2}\right\|_{2, p}\left\|\phi_{1}-\phi_{2}\right\|_{C^{2}},
\end{aligned}
$$

where, in view of Lemma 3.1, both constants $c$ depend only on $\|f\|_{C^{2}}$.

Now, from Lemma 5.1 one finally concludes the existence of a unique solution $v \in W^{2, p}\left(\Omega_{0}\right)$ to (34) satisfying the estimate (33).

\section{Analysis of the ODE}

In this final section we treat the ordinary differential equation for the free surface (27) with the boundary conditions (28). We rewrite the boundary value problem in the form

$$
\left\{\begin{array}{l}
-\varphi^{\prime \prime}+A(x) \varphi=H(x)+A(x) p_{0}, \quad-a<x<a \\
\varphi(-a)=\varphi(a)=f(a)
\end{array}\right.
$$

where

$$
A(x)=\frac{g \rho \cos \beta}{\sigma}\left(1+\phi^{\prime 2}\right)^{\frac{3}{2}}>0
$$

and

$$
H(x)=\frac{\alpha_{1}}{2 \sigma}\left(1+{\phi^{\prime}}^{2}\right)^{\frac{3}{2}}\left|\nabla u_{\phi}\right|^{2}+\alpha_{1}\left(1+\phi^{\prime 2}\right)^{\frac{3}{2}} \frac{g \rho \sin \beta}{\eta \sigma} v_{\phi}
$$

with $q_{\phi}=q \circ \mathcal{F}_{\phi}$. It is clear that the functions $u_{\phi}$ and $v_{\phi}$ which are defined in $\Omega_{\phi}$, are here evaluated at $y=\phi(x)$.

Lemma 6.1 Let $\phi \in C^{2}[-a, a]$ and $u, v \in W^{2, p}\left(\Omega_{0}\right)$ be given. Problem (36) admits a unique solution $\varphi \in C^{2}[-a, a]$. Moreover, if

$$
\|\phi\|_{C^{2}[-a, a]}+\|u\|_{2, p} \leqslant \varepsilon
$$

and $\varepsilon \ll 1$ is chosen sufficiently small, then the following estimate holds:

$$
\|\varphi-f(a)\|_{C^{0}[-a, a]}+\left\|\varphi^{\prime}\right\|_{C^{1}[-a, a]} \leqslant C\left(\delta+\delta \varepsilon+\varepsilon^{2}+(1+\varepsilon) \sin \beta_{0}\|v\|_{2, p}\right) .
$$

Proof. By classical results on ODEs it follows that (36) has a unique $C^{2}$ solution. In fact, the assumptions on $\phi, u$ and $v$, the geometrical properties of our two-dimensional domain and Sobolev's embedding theorem (recall that $p>2$ ) imply that $A$ and $H$ are continuous functions on $[-a, a]$, which together with the fact that $A$ is positive is enough to guarantee the result.

To obtain the estimates, observe that letting $\psi(x)=\varphi(x)-f(a)$ and taking (A2) into account, problem (36) transforms into

$$
\left\{\begin{array}{l}
-\psi^{\prime \prime}+A(x) \psi=H(x)+\delta A(x), \quad-a<x<a \\
\psi(-a)=\psi(a)=0 .
\end{array}\right.
$$


Hence, we have the estimate

$$
\|\psi\|_{C^{2}[-a, a]} \leqslant C\left(\|H\|_{C^{0}[-a, a]}+\delta\|A\|_{C^{0}[-a, a]}\right) .
$$

In order to estimate $H$, let us point out that

$$
\left\|\left.\left(\nabla u_{\phi}\right)\right|_{y=\phi(x)}\right\|_{C^{0}[-a, a]} \leqslant C\|u\|_{C^{1}} \max _{x \in[-a, a]}\left|\nabla \mathcal{F}_{\phi}^{2}\right|_{y=\phi(x)},
$$

where $\mathcal{F}_{\phi}=\left(\mathcal{F}_{\phi}^{1}, \mathcal{F}_{\phi}^{2}\right)$ is the mapping defined by (17). Therefore, in view of (37), Lemma 3.1 and Sobolev's embedding theorem, one obtains

$$
\left\|\left.\left(\nabla u_{\phi}\right)\right|_{y=\phi(x)}\right\|_{C^{0}[-a, a]} \leqslant C\|u\|_{2, p} \leqslant C \varepsilon .
$$

Now, one easily concludes that

$$
\|H\|_{C^{0}[-a, a]} \leqslant C\left(\varepsilon^{2}+(1+\varepsilon) \sin \beta_{0}\|v\|_{2, p}\right)
$$

and

$$
\|A\|_{C^{0}[-a, a]} \leqslant C(1+\varepsilon),
$$

where we have used (37) and the fact that $\varepsilon \ll 1$. The conclusion is now obvious.

LEMMA 6.2 Let $\phi_{1}, \phi_{2} \in C^{2}[-a, a]$ and $u_{1}, u_{2}, v_{1}, v_{2} \in W^{2, p}\left(\Omega_{0}\right)$ be given. If

$$
\left\|\phi_{i}\right\|_{C^{2}}+\left\|u_{i}\right\|_{2, p} \leqslant \varepsilon, \quad i=1,2
$$

and $\varepsilon \ll 1$ is chosen sufficiently small, then the following estimate holds:

$$
\left\|\varphi_{1}-\varphi_{2}\right\|_{C^{2}} \leqslant C\left(\left(\delta+\varepsilon+\varepsilon\left\|v_{1}\right\|_{2, p}\right)\left\|\phi_{1}-\phi_{2}\right\|_{C^{2}}+\varepsilon\left\|u_{1}-u_{2}\right\|_{2, p}+\sin \beta_{0}\left\|v_{1}-v_{2}\right\|_{2, p}\right),
$$

where $\varphi_{1}$ (respectively $\varphi_{2}$ ) is the unique solution to problem (36) corresponding to the data $\phi_{1}, u_{1}, v_{1}$ (respectively $\phi_{2}, u_{2}, v_{2}$ ).

Proof. Let $\psi_{i}(x)=\varphi_{i}(x)-f(a), i=1,2$ be two solutions and

$$
\psi(x)=\psi_{1}(x)-\psi_{2}(x)=\varphi_{1}(x)-\varphi_{2}(x) .
$$

We then have that

$$
-\psi^{\prime \prime}+A_{1}(x) \psi=H_{1}(x)-H_{2}(x)+\left(A_{1}(x)-A_{2}(x)\right)\left(\delta-\psi_{2}(x)\right)
$$

and consequently

$$
\|\psi\|_{C^{2}} \leqslant C\left[\left\|H_{1}-H_{2}\right\|_{C^{0}}+\left\|A_{1}-A_{2}\right\|_{C^{0}}\left\|\psi_{2}\right\|_{C^{2}}+\delta\left\|A_{1}-A_{2}\right\|_{C^{0}}\right] .
$$

Now

$$
A_{1}-A_{2}=C\left[\left(1+{\phi_{1}}^{\prime 2}\right)^{\frac{3}{2}}-\left(1+\phi_{2}{ }^{\prime 2}\right)^{\frac{3}{2}}\right]=f_{1}^{3}-f_{2}^{3}
$$


with $f_{i}=\sqrt[3]{C} \sqrt{1+{\phi_{i}}^{\prime 2}}$, hence

$$
\begin{aligned}
A_{1}-A_{2} & =\frac{f_{1}^{2}-f_{2}^{2}}{f_{1}+f_{2}}\left(f_{1}^{2}+f_{1} f_{2}+f_{2}^{2}\right) \\
& =\frac{\left(\phi_{1}{ }^{\prime}+\phi_{2}{ }^{\prime}\right)\left(\phi_{1}{ }^{\prime 2}+\phi_{1}{ }^{\prime} \phi_{2}{ }^{\prime}+\phi_{2}{ }^{2}\right)}{\left(1+{\phi_{1}}^{\prime 2}\right)^{\frac{1}{2}}+\left(1+\phi_{2}{ }^{\prime 2}\right)^{\frac{1}{2}}}\left(\phi_{1}{ }^{\prime}-\phi_{2}{ }^{\prime}\right)
\end{aligned}
$$

and

$$
\left\|A_{1}-A_{2}\right\|_{C^{0}} \leqslant C \varepsilon^{3}\left\|\phi_{1}-\phi_{2}\right\|_{C^{1}} .
$$

On the other hand, setting $u_{\phi_{i}}^{i}=u_{i} \circ \mathcal{F}_{\phi_{i}}, i=1,2$, one gets

$$
\begin{aligned}
& \left\|\left.\left(\nabla u_{\phi_{1}}^{1}\right)\right|_{y=\phi_{1}(x)}-\left.\left(\nabla u_{\phi_{2}}^{2}\right)\right|_{y=\phi_{2}(x)}\right\|_{C^{0}[-a, a]} \\
& \leqslant \\
& \quad C\left\|u_{1}-u_{2}\right\|_{C^{1}} \max _{x \in[-a, a]}\left|\nabla \mathcal{F}_{\phi_{1}}^{2}\right|_{y=\phi_{1}(x)} \\
& \quad+\left\|u_{2}\right\|_{C^{1}} \max _{x \in[-a, a]}\left|\left(\nabla \mathcal{F}_{\phi_{1}}^{2}\right)\right|_{y=\phi_{1}(x)}-\left.\left(\nabla \mathcal{F}_{\phi_{2}}^{2}\right)\right|_{y=\phi_{2}(x)} \mid .
\end{aligned}
$$

Observing that

$$
\left(\nabla \mathcal{F}_{\phi}\right)(x, \phi(x))=\left[\begin{array}{cc}
1 & 0 \\
r\left(z_{1}, f(a)\right) & s\left(z_{1}\right)
\end{array}\right]
$$

and taking estimates (35) of Lemma 5.2 into account, one obtains

$$
\max _{x \in[-a, a]}\left|\left(\nabla \mathcal{F}_{\phi_{1}}^{2}\right)\right|_{y=\phi_{1}(x)}-\left.\left(\nabla \mathcal{F}_{\phi_{2}}^{2}\right)\right|_{y=\phi_{2}(x)} \mid \leqslant C\left\|\phi_{1}-\phi_{2}\right\|_{C^{1}} .
$$

Therefore, for $\varepsilon \ll 1$ sufficiently small, one easily concludes that

$$
\begin{aligned}
\left\|H_{1}-H_{2}\right\|_{C^{0}} \leqslant & C\left(\left(1+\left\|v_{1}\right\|_{C^{0}}\right) \varepsilon^{2}\left\|\phi_{1}-\phi_{2}\right\|_{C^{1}}+\varepsilon\left\|u_{1}-u_{2}\right\|_{C^{1}}\right. \\
& \left.+\sin \beta_{0}\left\|v_{1}-v_{2}\right\|_{C^{0}}\right),
\end{aligned}
$$

which together with (42) and Sobolev's embedding theorem gives the desired estimate.

\section{Acknowledgements}

The authors would like to thank S. Nazarov and V. Solonnikov for useful suggestions and remarks concerning the paper. They are also grateful to the anonymous referee for the comments that helped improving the final version of the paper. The research of JMU is partially supported by CMUC/FCT and the research of JHV is partially supported by CMA/FCT.

\section{REFERENCES}

1. Bemelmans, J. On a free boundary problem for the stationary Navier-Stokes equations. Ann. Inst. Henri Poincaré 4, (1987) 517-547. 
2. Dunn, J. E. \& Fosdick, R. L. Thermodynamics, stability and boundedness of fluids of complexity 2 and fluids of second grade. Arch. Ration. Mech. Anal. 56, (1974) 191-252.

3. Grisvard, P. Elliptic Problems in Nonsmooth Domains, Monographs and Studies in Mathematics 24. Pitman, Boston (1985).

4. Hansen, E. B. \& Solonnikov, V. A. An existence theorem for Poiseuille flow with surface tension in an open channel. Math. Meth. Appl. Sci. 13, (1990) 23-30.

5. NAZAROV, S. \& PILECKAS, K. On noncompact free boundary problems for the plane stationary NavierStokes equations. J. Reine Angew. Math. 438, (1993) 103-141.

6. Pileckas, K., Sequeira, A., \& Videman, J. H. Steady flows of viscoelastic fluids in domains with outlets to infinity. J. Math. Fluid. Mech. 2, (2000) 185-218.

7. Pileckas, K. \& Solonnikov, V. A. On stationary Stokes and Navier-Stokes systems in an open infinite channel, I. Lit. Mat. Sb. 29, (1989) 90-108. II. Lit. Mat. Sb. 29, (1989) 347-367 (in Russian).

8. PUCHNACHOV, V. V. The plane stationary problem with a free boundary for the Navier-Stokes equations. Zh. Prikl. Mekh. Tekh. Fiz. 3, (1972) 91-102. English Translation. J. Appl. Mech. Techn. Phys. 13, (1972).

9. Rivlin, R. S. Solution of some problems in the exact theory of visco-elasticity. J. Ration. Mech. Anal. 5, (1956) 179-188.

10. Rivlin, R. S. \& ERICKSEN, J. L. Stress-deformation relations for isotropic materials. J. Ration. Mech. Anal. 4, (1955) 323-425.

11. Rodrigues, J. F. \& Urbano, J. M. Degenerate elliptic problems in a class of free domains. J. Math Pures Appl. 78, (1999) 819-840.

12. Sattinger, D. On the free surface of a viscous fluid. Proc. R. Soc. London A 349, (1976) 183-204.

13. Solonnikov, V. A. On the Stokes equations in domains with non-smooth boundaries and on viscous incompressible flow with a free surface. Collège de France Seminars 3, (1980/81) 340-423.

14. URBAno, J. M. On the mathematical analysis of a valley glacier model. In: CRETE (ed), Free Boundary Problems: Theory and Applications, Research Notes in Mathematics 409. pp. 237-245. Chapman \& Hall/CRC, Boca Raton, FL (1999).

15. URbano, J. M. \& Videman, J. H. A free domain problem for a viscoelastic fluid subject to a flux condition, in preparation.

16. Videman, J. H. Mathematical analysis of viscoelastic non-Newtonian fluids, PhD Thesis, Instituto Superior Técnico, Technical University of Lisbon, (1997). 